Published in final edited form as:

Mol Immunol. 2007 March ; 44(7): 1784-1792.

\title{
Initiation of $\mathrm{V}(\mathrm{D}) \mathrm{J}$ recombination in zebrafish (Danio rerio) ovaries
}

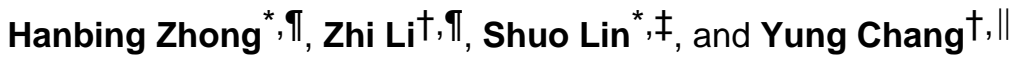 \\ * Center of Developmental Biology and Genetics, College of Life Sciences, Peking University, \\ Beijing 100871, P. R. CHINA \\ † Genomics, Evolution and Bioinformatics, School of Life Sciences, The Biodesign Institute at \\ Arizona State University, PO Box 875001, Tempe, AZ 85287-5001 \\ $\ddagger$ Department of Molecular, Cell, and Developmental Biology, University of California, Los Angeles, \\ California 90095, USA
}

\begin{abstract}
The assembly of mammalian antigen receptor genes is a lymphoid-specific process. However, rearranged immunoglobulin genes can also be recovered from non-lymphoid tissues of cartilaginous fish. This event, known as germline rearrangement, has been speculated to arise from recombinationactivating gene (RAG)-mediated recombination in germ cells. In this report, we demonstrate that zebrafish (Danio rerio) oocytes expressing high levels of RAG-RNA can readily initiate recombination cleavage at immunoglobulin gene loci, providing direct evidence for an ongoing process of attempted germline rearrangement in zebrafish ovaries. This attempted rearrangement is largely unproductive, yielding no accumulation of germline-joined immunoglobulin genes in zebrafish, which is consistent with their general absence in this species. Our data, therefore, substantiate the speculation that RAG might have been derived from a transposase, invading germ cells of ancient species, and later become a dedicated recombinase only expressed in developing lymphocytes.
\end{abstract}

\section{Keywords}

germline gene rearrangement; Ligation-mediated PCR; non-homologous end joining; recombination ends; $\mathrm{V}(\mathrm{D}) \mathrm{J}$ recombination

\section{Introduction}

The lymphoid-specific recombinases encoded by the recombination-activating gene 1 and 2 ( $R A G 1$ and $R A G 2$ ) catalyze a site-specific $\mathrm{V}(\mathrm{D}) \mathrm{J}$ recombination reaction. RAG1 and RAG2 proteins recognize recombination signal sequences (RSSs) and cleave the DNA substrates to generate two types of recombination intermediates: blunt-ended recombination signal ends (SEs) and covalently sealed hairpin coding ends (CEs) (Schatz, 2004). Signal and coding ends are resolved into signal joints (SJs) and coding joints (CJs), respectively, by non-homologous end joining (NHEJ) proteins, including Ku70, Ku80, XRCC4 and DNA ligase IV, as well as hairpin opening and overhang processing proteins, DNA-dependent protein kinase catalytic subunit (DNA-PKcs) and Artemis (Schatz, 2004).

$\mathbb{I}_{\text {These authors contributed equally to this work. }}$

"Correspondence should be addressed to Y.C. (yung.chang@asu.edu)

Publisher's Disclaimer: This is a PDF file of an unedited manuscript that has been accepted for publication. As a service to our customers we are providing this early version of the manuscript. The manuscript will undergo copyediting, typesetting, and review of the resulting proof before it is published in its final citable form. Please note that during the production process errorsmaybe discovered which could affect the content, and all legal disclaimers that apply to the journal pertain. 
RAG1/2 genes are highly conserved in jawed vertebrates and may have been derived from the horizontal acquisition of transposons by primitive jawed vertebrates (Hiom et al., 1998; Kapitonov and Jurka, 2005; Schatz, 2004). Alternatively, a recent report suggests that the RAGs may have originated by a more typical vertical evolutionary process, following the event that the prototypes of RAG1/2 as transposase components inserted into the genome of an earlier ancestor of the jawed vertebrates (Fugmann et al., 2006). Regardless the précise details of the origin of the RAG genes, the central role of RAGs in V(D)J recombination as well as creating adaptive immune system in jawed vertebrates remains unaltered. In particular, the coexpression of RAG1/2 is directly associated with recombination activity in developing lymphocytes and is responsible for creating the diverse antigen receptor repertoires of the adaptive immune system (Schatz, 2004). However, rearranged immunoglobulin genes can also be recovered from the nonlymphoid cells of cartilaginous fish, including germ cells. These rearranged genes, known as germline-joined genes, represent heritable specificity and may offer innate protection to fish embryos likely to be surrounded by pathogens in the environment (Cannon et al., 2004; Litman et al., 1999; Rumfelt et al., 2001).

RAG1 transcripts were found in zebrafish ovaries, implicating the potential role of RAG1/2 in germline rearrangement (Willett et al., 1997). However, direct evidence for the role of RAG1/2 expression in mediating germline rearrangement is still lacking. In this study, we demonstrate that zebrafish oocytes expressing RAG1/2 can initiate recombination cleavage at immunoglobulin genes, and thus provide direct evidence for ongoing recombination initiated in germ cells. The attempted recombination in zebrafish oocytes, unlike primary lymphoid tissues, fails to produce successfully recombined products and thereby offers no germlinejoined immunoglobulin genes for the zebrafish offspring. These data are discussed in the context of evolution of immunoglobulin gene assembly in jawed vertebrates.

\section{Materials \& Methods}

\subsection{Animals and DNA preparation}

Adult zebrafish (Danio rerio) were raised and maintained in a zebrafish facility (Aquatic EcoSystems, Inc. FL) at $28.5^{\circ} \mathrm{C}$ with a photoperiod of $14 \mathrm{hrs} \mathrm{light} \mathrm{and} 10 \mathrm{hrs}$ dark, according to the established guideline (Westerfield, 1995). All animal protocols were conducted under the supervision and approval of the ASU IACUC committee. Kidney, ovary, testis, heart and spleen were taken from adult zebrafish after euthanasia. Single cell suspensions of these tissues were prepared by homogenization with frosted slides. Preparation of ovarian cells required that cell clumps be dispersed manually under the dissecting microscope with fine forceps. A dejellying procedure was performed with $10 \mathrm{mM}$ DTT/PBS at room temperature for $20 \mathrm{~min}$ to disrupt big eggs. To obtain intact chromosomal DNA, cells were embedded in agarose plugs, which were then subjected to deproteinization with proteinase K (Chang and Brown, 1999).

\subsection{RNA preparation and RT-PCR}

Total RNA was extracted from homogenized tissue using TRIzol reagent (Invitrogen), according to the manufacturer's instructions. Genomic DNA was removed by treating samples with RNase-free DNase (Roche). RNA was reverse transcribed (RT) into cDNA using the Moloney murine leukemia virus reverse transcriptase (Promega) incubated at $37{ }^{\circ} \mathrm{C}$ for $2 \mathrm{hrs}$ with $1 \mathrm{mM}$ of random hexamers and $5 \mathrm{mM}$ nucleotides. The same amount of RNA was also set up for the similar RT reaction, except without the addition of reverse transcriptase, which serves as a RT control for assessing genomic DNA contamination. A serially diluted cDNAs (1, 1:3, 1:9), as well as RT control samples, were amplified for RAG1 and RAG2 and $\beta$-actin using primers listed in Table 1. $\beta$-actin served as an internal control for input cDNA. The RAG1 primers are complementary to the sequences located at two different exons such that PCR products of RAG1 cDNA (at size of 888bps) can be readily distinguished from the one 
amplified from contaminated genomic DNA (Willett et al., 1997). Although RAG2 PCR products amplified from cDNA or genomic DNA exhibit the same size because of its single exon structure, PCR on the RT controls should tell the presence or absence of genomic DNA.

\subsection{Whole mount in-situ hybridization}

The wild type AB strain was used in this study. Embryos were staged as described by Kimmel et al. (Kimmel et al., 1995).

Dissected ovaries from adult females and testes from adult males were sliced into pieces of approximately $2 \mathrm{~mm}$ and fixed in $4 \%$ paraformaldehyde/PBS overnight. RNA whole mount in situ hybridization was performed as described for whole zebrafish embryos (Westerfield, 1995). Digoxigenin-labeled antisense and sense RNA probes were generated in vitro using the zebrafish RAG1 or RAG2 cDNA as templates. After staining, ovaries and testes were sectioned at $5 \mathrm{~mm}$ in paraffin, as displayed in Figure $1 \mathrm{~F}-1 \mathrm{G}$ and $1 \mathrm{~J}-1 \mathrm{~K}$. Section $1 \mathrm{~J}$ and $1 \mathrm{~K}$ were further counterstained with nuclear fast red to stain nuclei, as shown in Figure 1L-1M (Leubitz and Savage, 1984).

Images of zebrafish ovaries, testes and embryos were acquired using Zeiss Axioplan 2 equipped with AxioCam digital camera and OpenLab (Improvision, Lexington, MA) software and edited with Photoshop 7.0 (Adobe Systems Incorporated, San, Jose, CA).

\subsection{LM-PCR}

Ligation-mediated PCR (LM-PCR) was used to analyze double-stranded DNA breaks (DSBs), generated from a site-specific recombination excision (Suppl. Fig. 1A). According to the procedure reported by Roth et al (Roth et al., 1993), an artificial linker made by annealing the two primers 25 and 116 (listed in Table 1) can be ligated to the blunted SEs using T4 DNA ligase (Boehringer Mannheim). To detect staggered recombination ends, DNA plugs were pretreated with T4-DNA polymerase (T4-DNA-POL) then ligated to the linker. Ligated DNA was then amplified for $\mathrm{J}_{\mathrm{H}^{-}}$or $\mathrm{J}_{\mathrm{L}}$-SEs and CEs, as well as $\mathrm{DJ}_{\mathrm{H}^{-}}$or $\mathrm{VJ}_{\mathrm{L}}-\mathrm{CJ}_{\mathrm{S}}$ and $\mathrm{SJ}$ s using primer pairs listed in Table 1 and 2. Locations of these primers were presented in supplemental Figure $1 \mathrm{~B}$.

\section{Results}

\subsection{Expression of RAG1 and RAG2 in gonad tissues}

RAG1 transcripts have been reported to be present in the zebrafish ovaries (Willett et al., 1997). To further understand the significance of this finding, we wanted to determine whether both RAG1 and RAG2 RNA are present in gonad tissues. The expression of RAG1 and RAG2 in various tissues was assessed by reverse-transcription PCR (RT-PCR). Before subjecting to reverse transcription, RNA samples were treated with DNase to remove genomic DNA. As shown in Figure 1A, both RAG1 and RAG2 transcripts were readily detectable in the ovary and the testis at a level comparable to the one in the kidney, the primary lymphoid organ in adult zebrafish (Traver, 2004) (Fig. 1A lane 2-4 to 6-8 and 10-12). As expected, no RAG1/2 transcripts were found in the heart, a non-lymphocyte tissue as a negative control (Fig. 1A, lane 14-16), neither in the RT controls, i.e., without prior reverse transcription (Fig. 1A, lane 1, 5, 9 and 13). Thus, the RAG1/2 RT-PCR products detected clearly indicate the presence of RAG1/2 transcripts, but not contaminated genomic DNA, in the ovary and the testis,. Our finding confirms the previous study, and shows the co-expression of RAG1/2 in both zebrafish ovary and testis.

The localizations of RAG1/2 transcripts in the ovary and testis were further analyzed by in situ hybridization. Both RAG1 and 2 transcripts were identified in early developmental stages 
of oocytes by in situ hybridization according to the morphology of the oocytes (Fig. 1B-1G) (Selman et al., 1993). Although RAG2 could be detected in the rounded cells in the testis (Fig. $1 \mathrm{I}, 1 \mathrm{~K}$ and $1 \mathrm{M}$ ), probably representing spermatogonia and primary spermatocytes (Leung et al., 2005), RAG1 was not found in these cells, but rather appear non-specifically (data not shown). The specificity of these RAG-expressing cells was clearly demonstrated by their reactivity with only anti-sense (Fig. 1C, 1E, 1G, 1I, 1K and 1M), but not sense RAG probes (Fig. 1B, 1D, 1F, 1H, 1J and 1L), which are similar to the profile observed in our positive control, thymus of a whole embryo (Fig. 1N-1U). These results indicate that zebrafish oocytes concurrently express RAG1 and RAG2, a prerequisite for recombination activity in developing lymphocytes.

\subsection{Presence of recombination ends in the ovaries}

To determine whether expressed RAG1/2 can contribute to germline rearrangement, we analyzed recombination intermediates from zebrafish germ cells. These intermediates are transiently produced and are indicative of ongoing recombination (Roth et al., 1993).

Specifically, we used ligation-mediated PCR (LM-PCR) to amplify recombination SEs made at immunoglobulin light chain (IgL) gene locus, i.e., $\mathrm{J}_{\mathrm{L}}$-SEs (Supplemental Fig. 1A and 1B), according to a well-established protocol (Roth et al., 1993; Schlissel et al., 1993). The immunoglobulin gene loci analyzed in this study were shown in Supplemental Figure 1B, including primers utilized in PCR amplification of recombination ends and joints (Table 1 and 2). The kidney containing recombination-active cells was included as our positive control for end detection. After semi-nested PCR amplification, $\mathrm{J}_{\mathrm{L}}-\mathrm{SEs}$ could be readily detected in the kidney and the ovary, but not the testis (Fig. 2A). Thus, we provide the first evidence of attempted IgL gene rearrangement in the ovary, a non-lymphoid tissue.

Next, we examined recombination CEs using a modified LM-PCR. The CEs present in the murine lymphocyte precursors usually contain 3'-overhang, and their detection by LM-PCR requires pre-treatment of DNA molecules with T4-DNA polymerase (T4-DNA POL) to flush the ends before their ligation to the linker (Schlissel, 1998). Again, the kidney was included in the analysis. To compare the recombination activity between ovary and kidney, we used semiquantitative LM-PCR and PCR reactions followed by Southern blot to assess the levels of recombination ends and joints, respectively. As described in our recent study (accompanied manuscript), if DNA-plugs were pre-treated with T4-DNA-POL, the detection of recombination SEs and CEs was greatly enhanced, indicating that both SEs and CEs made by zebrafish lymphoid tissues (i.e., kidney) were staggered. As shown in Figure 2B, upon T4DNA-POL treatment, both $\mathrm{J}_{\mathrm{H}^{-}} \mathrm{SEs}$ and $\mathrm{J}_{\mathrm{H}^{-}}$-CEs made at the immunoglobulin heavy chain locus (IgH) could be detected in the ovary, at a level comparable to that in the kidney.

Interestingly, unlike the distinct band of SEs amplified by the semi-nested LM-PCR (Fig. 2A), the primary LM-PCR products of SEs, as well as CEs, show variation in size (Fig. 2B, lane 3 and 7) and more than one band appear after LM-PCR amplification of diluted DNA (Fig. 2B, lane 4 and 8). This finding indicates that these SEs are being modified with either nucleotide additions or deletions. In the semi-nested PCR, however, only restricted number of blunt SEs could be amplified since these DNA samples were not pre-treated with T4-DNA POL. To further characterize the structure of SEs, we performed the sequence analyses on individual LM-PCR products of SEs without prior T4-DNA-POL treatment. As summarized in Table 3, SEs made in the ovary suffer from nucleotide deletions, resembling our finding in the sequence analyses on kidney SEs (accompanied manuscript). These data indicate that the V(D)J recombination excision is initiated at the ovary immunoglobulin gene loci, generating recombination intermediates that bear the features similar to those produced by kidney.

We also analyzed the recombination status in zebrafish testis. Although RAG1/2 transcripts were detectable (Fig. 1A), no recombination ends $\left(\mathrm{J}_{\mathrm{H}^{-}} \mathrm{SEs}\right.$ and $\left.\mathrm{J}_{\mathrm{H}^{-}} \mathrm{CEs}\right)$ could be recovered 
from the testis even after T4-DNA-POL treatment (Fig. 3C). These findings indicate that oocytes are the primary site for the attempted immunoglobulin germline rearrangement in zebrafish.

\subsection{Few rearranged products accumulated in zebrafish ovaries}

Despite a comparable level of recombination cleavage initiated in the kidney and ovary, only a few SJs $\left(\mathrm{DJ}_{\mathrm{H}^{-}}-\mathrm{SJ}\right.$ s, $\left.\mathrm{VJ}_{\mathrm{L}}-\mathrm{SJs}\right)$ and virtually no $\mathrm{CJs}\left(\mathrm{DJ}_{\mathrm{H}^{-}} \mathrm{CJ}\right.$,, $\left.\mathrm{VJ}_{\mathrm{L}}-\mathrm{CJ} \mathrm{s}\right)$ could be recovered from the ovary (Fig. 3A and 3B). When the ratio of joints to ends (SJs/SEs and CJs/CEs) under the T4-DNA-POL treated condition was compared between the ovary and kidney, it is apparent that the ovary has much lower activity in resolving recombination ends than the kidney (Fig. 3C). The presence of mature B cells in the kidney but not the ovary may account for the different levels of CJs in these two tissues. On the other hand, the similar levels of recombination ends present in the kidney and ovary imply that these two tissues have a similar number of recombination-active cells, and yet the ovary makes a much lower level of SJs, which are the recombination by-products transiently produced in recombination-active cells. We further performed a semi-nested PCR on DNA samples without prior treatment with T4-DNA-POL to recover blunt SEs and few SJs since both SEs and SJs were found at comparable but very low levels in the ovary without prior T4-DNA-POL treatment (Fig. 3A and 3B, lane 5 and 6). As shown in Figure $4 \mathrm{~A}$ and $4 \mathrm{~B}$, while both $\mathrm{J}_{\mathrm{H}}-\mathrm{SEs}$ and $\mathrm{J}_{\mathrm{L}}-\mathrm{SEs}$ could be readily detected in the ovary and kidney, a lower level of $\mathrm{DJ}_{\mathrm{H}^{-}}-\mathrm{SJs}$ and $\mathrm{VJ}_{\mathrm{L}}-\mathrm{SJs}$ were recovered from the ovary than the kidney, which is consistent with the results from the primary PCR followed by Southern blot analyses (Fig. 3A and 3B).

Although we cannot completely rule out the possibility that the recombination ends detected in the ovary may result from contaminated blood-borne cells, the fact that no coding joints are recoverable reflects a unique feature of recombination-active cells in the ovary, i.e., attempted but incomplete recombination, making the contamination scenario very unlikely. The attempted recombination without completion raises a question as to whether the ovary is equipped with functional NHEJ. It has been well established that a defect in any one of the NHEJ components not only reduces the recombination efficiency, but also impairs the fidelity of the rearranged joints (Lieber et al., 1988; Taccioli et al., 1993; Zhu et al., 1996). Thus, by analyzing the junctional structures of the few SJs recovered from zebrafish ovaries, we can determine whether proficient or deficient NHEJ machinery is in place. To assess the joining fidelity of amplified SJs, we treated the amplified PCR products of $\mathrm{VJ}_{\mathrm{L}}-\mathrm{SJ}$ s with ApaL1, since the precise joining of two SEs, i.e., V-SEs and J-SEs, should create an ApaL1 restriction site. As shown in Figure $4 \mathrm{C}$, the $\mathrm{VJ}_{\mathrm{L}}$-SJs made in both ovary and kidney were susceptible to ApaL1 digestion, as reflected by the downshifted bands. Thus, the ovary can make perfect SJs and, based on sequence analyses, recovered SJs contain normal junctions (data not shown). These oocytes are likely to have intact but inefficient NHEJ machinery, which may allow a few SEs to be joined normally but leave the majority of SEs and probably all CEs unresolved.

Taken together, these results indicate that the ovary can initiate recombination cleavage at both $\mathrm{IgH}$ and IgL loci, and make a small amount of SJs, but apparently fails to form CJs. Our data suggest that recombination-active oocytes have an intrinsic inability in end resolution, which may be due to a lack of NHEJ components. Alternatively, inefficient NHEJ activity combined with a short life span of recombination-active oocytes during oogenesis may preclude the accumulation of joints to a detectable level.

\section{Discussion}

RAG1 transcript has been reported to be present in zebrafish ovary and RAG2 in Xenopus oocytes (Greenhalgh et al., 1993; Willett et al., 1997). The RT-PCR analyses in this study further confirm the presence of both RAG1 and RAG2 transcripts in the zebrafish ovary, as 
well as showing their existence in the testis. Our in situ hybridization analysis provides unequivocal evidence for RAG1/2 expression in developing oocytes of zebrafish (Fig. 1B-1G). RAG expression outside the lymphoid tissues raises two interesting questions: 1) what confers the RAG expression in the non-lymphoid tissues: cis-elements, trans-factors or both; and 2) what is the functional significance of RAG expression in the non-lymphoid tissues?

The RAG expression in zebrafish and Xenopus oocytes may result from global transcriptional activation during the diplotene phase of meiosis. In this phase, the chromosome is highly decondensed with many active transcription units, known as lampbrush chromosomes, which are present in amphibian, avian and fish oocytes, but not in mammalian counterparts (Macgregor, 1987). It is conceivable that the active transcription status in oocytes may also confer the immunoglobulin gene loci accessible to the recombinases. Interestingly, in cartilaginous fish where germline rearrangement is prevalent, RAG1 expression was also observed in gonad tissues, as well as other tissues, reflecting less stringent control over RAG1 transcription (Miracle et al., 2001). In addition, germline-rearranged products have also been identified in channel fish as well as certain avian species (Reynaud et al., 1989; VenturaHolman and Lobb, 2002). It is therefore reasonable that the active transcription status in the developing oocytes of fish and birds may fulfill the two prerequisites of V(D)J recombination initiation: RAG expression and immunoglobulin gene accessibility. The expression of RAG1/2 in germ cells may then be responsible for germline rearrangement. Our data of recombination ends present in the ovary provide direct evidence to support this speculation and reveal that the germline rearrangement is an ongoing process (at least in zebrafish) rather than an ancient event.

The fact that germline joining is restricted to IgH and IgL in shark, but not found in T cell receptor genes, suggests an association of germline joining with the multi-cluster organization of immunoglobulin genes (Cannon et al., 2004). However, our demonstration of recombination initiation at both $\mathrm{IgH}$ and $\operatorname{IgL}$ in the zebrafish ovaries clearly indicates that the multi-cluster organization is not a prerequisite, because the zebrafish IgH locus adopts a translocon configuration, in which $\mathrm{V}, \mathrm{D}$, and $\mathbf{J}$ gene segments are arranged in arrays, analogous to those seen in mammals (Cannon et al., 2004).

Although recombination SEs and CEs could be readily detected in the ovary at levels comparable to those in the kidney, only a few SJs and virtually no CJs could be recovered from the ovary. The presence of opened CEs in both kidney and ovary implies that these tissues are competent in processing hairpin CEs. The ovary, on the other hand, is not able to resolve all recombination ends into joints, presumably due to its inefficient NHEJ machinery. This speculation is consistent with several studies in Xenopus and mouse oocytes, which lack the activity of NHEJ factors until the later stages of egg development or the embryonic stage respectively (Fiorenza et al., 2001; Goedecke et al., 1992). Unlike somatic cells, mature eggs and embryos, double strand DNA breaks in developing oocytes are resolved predominantly by a homologous recombination (HR) pathway. It is possible that the meiotic recombination during oogenesis, which primarily occurs in stage I oocytes, suppresses the NHEJ machinery to ensure genome integrity since HR is a high-fidelity and conservative recombination process whereas NHEJ is an error-prone pathway. Therefore, while RAG1/2-expressing cells in zebrafish ovary can actively initiate immunoglobulin gene rearrangement, they fail to make $\mathrm{CJ}$, and thus are incapable of establishing pre-assembled immunoglobulin genes. Alternatively, the life span of RAG-expressing oocytes may be too short to allow an accumulation of rearranged joints in the oocytes to be detectable. Regardless of the underlying mechanisms, the lack of coding joint detection is consistent with the non-rearranged configuration of the $\mathrm{J}_{\mathrm{H}}$ locus reported recently by Danilova et al (Danilova et al., 2005). 
$\mathrm{V}$ (D)J recombination may have evolved from transposition, a hypothesis proposed even before the discovery of RAG1/2, because of the similarities of RSS with the inverted repeats found at the termini of transposons (Sakano et al., 1979). This hypothesis gained further support by the intron-less and compact organization of RAG1 and RAG2 genes, and in vitro demonstrations of core RAG1/2-mediated transposition (Agrawal et al., 1998; Hiom et al., 1998; Oettinger et al., 1990; Schatz et al., 1989). Finally, Kapitonov and Jurka provided direct structural evidence to prove that core RAG1 and RSS were derived from ancient DNA transposons of the Transib superfamily (Kapitonov and Jurka, 2005). During the horizontal transfer of this RAG transposon into primitive jawed vertebrates or even earlier species because of recent demonstration of RAG1/2 homolog in sea uchin (Fugmann et al., 2006), it is germ cells that might have initially acquired and passed this transposon onto future generations. Subsequently, the RAG-transposon might have evolved into present-day recombination machinery by physically dissociating the enzyme genes from their target genes to impose better control over potentially harmful recombination.

The fact of few or no germline joints recoverable from the zebrafish ovaries implies that germline rearrangement may have undergone involution from lower to higher vertebrates. Several lines of evidence support this hypothesis. First, bony fish have significantly fewer germline-joined genes compared to cartilaginous fish; germline-joined horned shark $\operatorname{IgH}$ comprise $50 \%$ of the clusters analyzed whereas only one germline VDJ joint has been identified so far in catfish (Ghaffari and Lobb, 1999; Litman et al., 1999; Ventura-Holman and Lobb, 2002). Second, the expression of RAG1/2 in germ cells appears to become extinct during evolution, e.g., both RAG1 and 2 transcripts are present in zebrafish, but only RAG2 is found in Xenopus oocytes, and neither is seen in adult mammalian germ cells (Greenhalgh et al., 1993; Schatz, 2004). Finally, the innate protection offered to embryos by pre-assembled antigen receptors may become less important from lower vertebrates to mammals because of increasingly better protection offered by maternal transfer of abundant antibodies to eggs or embryos, as well as the presence of a uterus (Gabriel et al., 2005; Swain et al., 2006). Therefore, the detected recombination activity in zebrafish germ cells may represent an evolutionary relic from when RAG proteins might have functioned as a transposase and later became a dedicated recombinase. On the other hand, the attempted recombination may lead to the formation of nonstandard rearranged products in zebrafish oocytes, which could serve to diversify immunoglobulin gene loci. This scenario will be tested in our future studies.

\section{Supplementary Material}

Refer to Web version on PubMed Central for supplementary material.

\section{Acknowledgement}

We thank Drs. J. Wilson-Rawls and D. Chandler for their advice in preparing single cell suspension from ovary. We thank S. Kumar and M. Gellert, as well as members of Chang's laboratory for critical reading of the manuscript. This work was partially supported by NCI (CA073857 to Y.C.) and NIH (DK054508 to S. L.). H.Z. is supported in part by funding from State Key Fundamental Research of China (G2000016109).

\section{Abbreviation}

CEs, coding ends; CJs, coding joints; DSBs, double strand DNA breaks; LM-PCR, Ligationmediated PCR; NHEJ, Non-homologous end joining; SEs, signal ends; SJs, signal joints.

\section{References}

Agrawal A, Eastman Q, Shatz D. Transposition mediated by RAG1 and RAG2 and its implications for the evolution of the immune system. Nature 1998;394:744-751. [PubMed: 9723614] 
Cannon JP, Haire RN, Rast JP, Litman GW. The phylogenetic orgins of the antigen-binding receptors and somatic diversification mechanisms. Immunol. Rev 2004;200

Chang Y, Brown ML. Formation of coding joints in V(D)J recombination-inducible severe combined immune deficient pre-B cell lines. Proc. Natl. Acad. Sci. USA 1999;96:191-196. [PubMed: 9874794]

Danilova N, Bussmann J, Jekosch K, Steiner LA. The immunoglobulin heavy-chain locus in zebrafish: identification and expression of a previously unknown isotype, immunoglobulin z. Nature Immunology 2005;6:295-302. [PubMed: 15685175]

Fiorenza MT, Bevilacqua A, Bevilacqua S, Mangia F. Growing dictyate oocytes, but not early preimplantation embryos, of the mouse display high levels of DNA homologous recombination by single-strand annealing and lack DNA nonhomologous end joining. Dev Biol 2001;233:214-24. [PubMed: 11319870]

Fugmann SD, Messier C, Novack LA, Cameron RA, Rast JP. An ancient evolutionary origin of the Rag1/2 gene locus. Proc Natl Acad Sci U S A 2006;103:3728-33. [PubMed: 16505374]

Gabriel S, Geldhof P, Phiri IK, Cornillie P, Goddeeris BM, Vercruysse J. Placental transfer of immunoglobulins in cattle infected with Schistosoma mattheei. Vet Immunol Immunopathol 2005;104:265-72. [PubMed: 15734547]

Ghaffari SH, Lobb CJ. Structure and genomic organization of a second cluster of immunoglobulin heavy chain gene segments in the channel catfish. J. Immunol 1999;162:1519-1529. [PubMed: 9973409]

Goedecke W, Vielmetter W, Pfeiffer P. Activation of a system for the joining of nonhomologous DNA ends during Xenopus egg maturation. Mol Cell Biol 1992;12:811-6. [PubMed: 1732745]

Greenhalgh P, Olesen CE, Steiner LA. Characterization and expression of recombination activating genes (RAG-1 and RAG-2) in Xenopus laevis. J. Immunol 1993;151:3100-3110. [PubMed: 8376769]

Hiom K, Melek M, Gellert M. DNA transposition by the RAG1 and RAG2 proteins: a possible source of oncogenic translocations. Cell 1998;94:463-470. [PubMed: 9727489]

Kapitonov VV, Jurka J. RAG1 core and V(D)J recombination signal sequences were derived from Transib transposons. PLoS Biol 2005;3:e181. [PubMed: 15898832]

Kimmel CB, Ballard WW, Kimmel SR, Ullmann B, Schilling TF. Stages of embryonic development of the zebrafish. Dev Dyn 1995;203:253-310. [PubMed: 8589427]

Leubitz SS, Savage RA. Sensitivity of picroindigocarmine/nuclear fast red (PIC/NF) stain for detection spermatozoa: a serial dilution study of human ejaculate. Am. J. Clin. Pathol 1984;81:90-93. [PubMed: 6197878]

Leung AY, Leung JC, Chan LY, Ma ES, Kwan TT, Lai KN, Meng A, Liang R. Proliferating cell nuclear antigen (PCNA) as a proliferative marker during embryonic and adult zebrafish hematopoiesis. Histochem Cell Biol 2005;124:105-11. [PubMed: 16028068]

Lieber MR, Hesse JE, Lewis S, Bosma GC, Rosenberg N, Mizuuchi K, Bosma MJ, Gellert M. The defect in murine severe combined immune deficiency: joining of signal sequences but not coding segments in V(D)J recombination. Cell 1988;55:7-16. [PubMed: 3167977]

Litman GW, Anderson MK, Rast JP. Evolution of antigen binding receptors. Ann. Rev. Immunol 1999;17:109-147. [PubMed: 10358755]

Macgregor HC. Lampbrush chromosomes. J Cell Sci 1987;88(Pt 1):7-9. [PubMed: 3327866]

Miracle AL, Anderson MK, Litman RT, Walsh CJ, Luer CA, Rothenberg EV, Litman GW. Complex expression patterns of lymphocyte-specific genes during the development of cartilaginous fish implicate unique lymphoid tissues in generating an immune repertoire. Int. Immunol 2001;13:567580. [PubMed: 11282996]

Oettinger MA, Schatz DG, Gorka C, Baltimore D. RAG-1 and RAG-2, adjacent genes that synergistically activate V(D)J recombination. Science 1990;248:1517-1523. [PubMed: 2360047]

Reynaud CA, Dahan A, Anquez V, Weill JC. Somatic hyperconversion diversifies the single Vh gene of the chicken with a high incidence in the D region. Cell 1989;59:171-83. [PubMed: 2507167]

Roth DB, Zhu C, Gellert M. Characterization of broken DNA molecules associated with V(D)J recombination. Pro. Natl. Acad. Sci. USA 1993;90:10788-10792.

Rumfelt LL, Avila D, Diaz M, Bartl S, McKinney EC, Flajnik MF. A shark antibody heavy chain encoded by a nonsomatically rearranged VDJ is preferrentially expressed in early development and is convergent with mammalian IgG. Pro. Natl. Acad. Sci. USA 2001;98:1775-1780. 
Sakano H, Huppi K, Heinrich G, Tonegawa S. Sequences at the somatic recombination sites of immunoglobulin light-chain genes. Nature 1979;280:288-94. [PubMed: 111144]

Schatz DG. Antigen receptor genes and the evolution of a recombinase. Semin. Immunol 2004;16:245256. [PubMed: 15522623]

Schatz DG, Oettinger MA, Baltimore D. The V(D)J recombination activating gene (RAG-1). Cell 1989;59:1035-1048. [PubMed: 2598259]

Schlissel M, Constantinescu A, Morrow T, Baxter M, Peng A. Double-strand signal sequence breaks in $\mathrm{V}(\mathrm{D}) \mathrm{J}$ recombination are blunt, 5'-phosphorylated, RAG-dependent, and cell cylce regulated. Genes Dev 1993;7

Schlissel MS. Structure of nonhairpin coding-end DNA breaks in cells undergoing V(D)J recombination. Mol Cell Biol 1998;18:2029-37. [PubMed: 9528775]

Selman K, Wallace RA, Sarka A, Qi X. Stage of oocyte development in zebrafish brachydanio rerio. J. Morphol 1993;218:203-224.

Swain P, Dash S, Bal J, Routray P, Sahoo PK, Sahoo SK, Saurabh S, Gupta SD, Meher PK. Passive transfer of maternal antibodies and their existence in eggs, larvae and fry of Indian major carp, Labeo rohita (Ham.). Fish Shellfish Immunol 2006;20:519-27. [PubMed: 16157486]

Taccioli GE, Rathbun G, Oltz E, Stamato T, Jeggo PA, Alt FW. Impairment of V(D)J recombination in double-strand break repair mutants. Science 1993;260:207-10. [PubMed: 8469973]

Traver D. Cellular Dissection of Zebrafish hematopoiesis. Methods in Cell Biology 2004;76:127-149. [PubMed: 15602875]

Ventura-Holman T, Lobb CJ. Structural organization of the immunoglobulin heavy chain locus in the channel catfish: the IgH locus represents a composite of two gene clusters. Mol Immunol 2002;38:557-64. [PubMed: 11750657]

Westerfield, M. The Zebrafish Book: A Guid for the Laboratory Use of Zebrafish (Brachydanio rerio). University of Oregon Press; Eugene: 1995.

Willett CE, Cherry JJ, Steiner LA. Characterization and expression of the recombination activating genes (rag1 and rag2) of zebrafish. Immunogenetics 1997;45:394-404. [PubMed: 9089097]

Zhu C, Bogue MA, Lim D-S, Hasty P, Roth DB. Ku86-deficient mice exhibit severe combined immunodeficiency and defective processing of V(D)J recombination intermediates. Cell 1996;86:379-389. [PubMed: 8756720] 

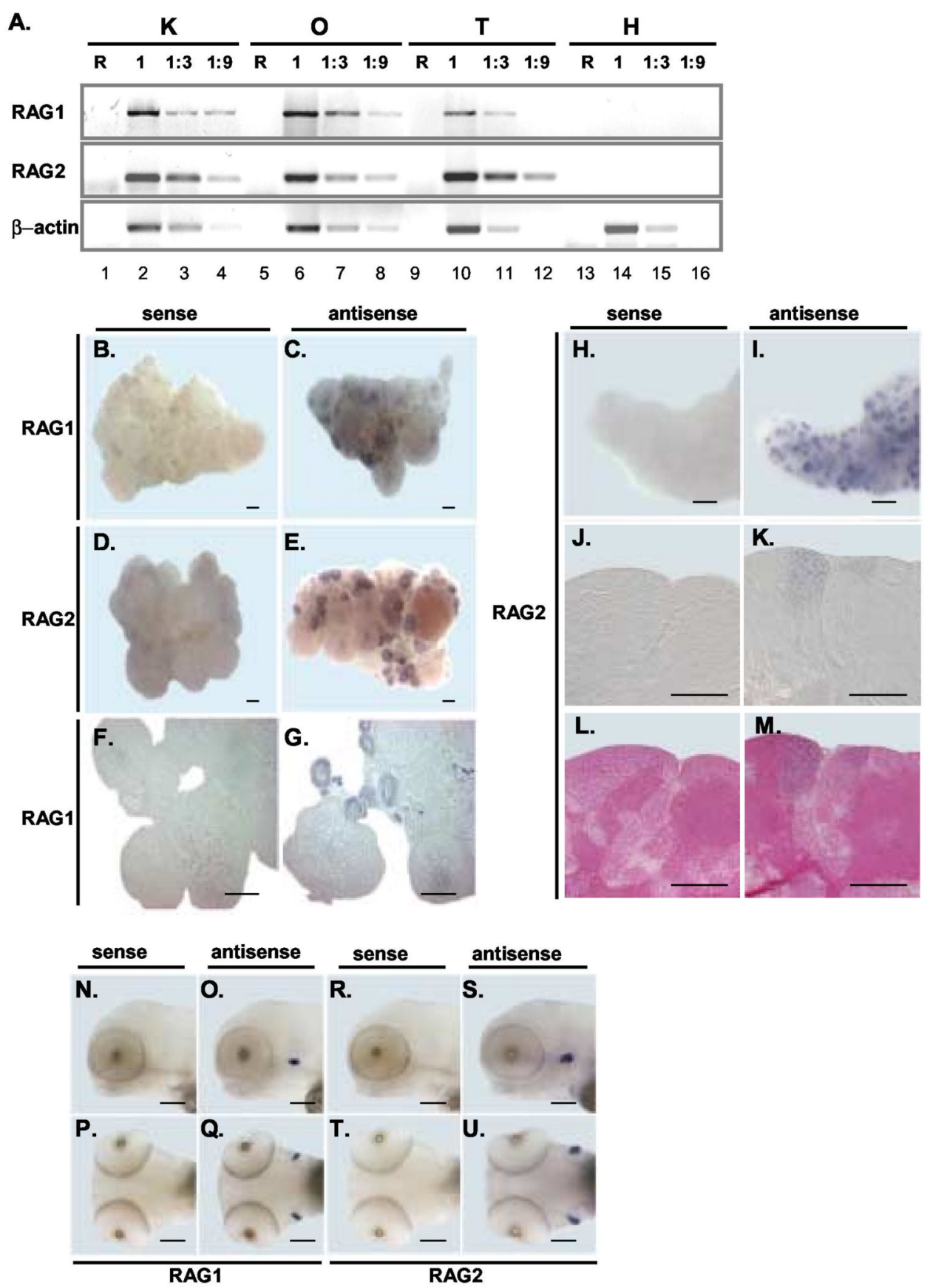

Fig. 1.

Expression of RAG1 and RAG2 in gonad tissues. A. Semi-quantitative RT-PCR analyses of RAG1 and RAG2 transcripts (serial 3-fold dilutions of input cDNA) in various adult tissues, kidney $(\mathrm{K})$, ovary $(\mathrm{O})$, testis $(\mathrm{T})$ and heart $(\mathrm{H}) . \beta$-actin serves as a loading control for the input cDNA used in each reaction. RNA without reverse transcription $(\mathrm{R})$ was used as a negative control. B-M. Expression of RAG1/2 in ovary and RAG2 in testis detected by whole mount in situ hybridization. B-G. RAG1 and RAG2 mRNA in stage I/II oocytes. B. Lack of RAG1 hybridization with a sense RAG1 probe. C. RAG1 mRNA revealed by an anti-sense RAG1 probe; D. Sense control of RAG2, E. Revelation of RAG2 RNA by an anti-sense RAG2 probe; F. Section of samples from (B); G. Section of samples from (C). H-M. Whole mount in situ 
hybridization of RAG2 mRNA in testis. H. Sense control of RAG2; I. Revelation of RAG2 by an anti-sense RAG2 probe; J-M. Paraffin sections of samples $\mathrm{H}$ and I, i.e., J and L from $\mathrm{H}$, and $\mathrm{K}$ and $\mathrm{M}$ from I; L-M. Sections (J, K) counterstained with nuclear fast red to show nuclei (Leubitz and Savage, 1984). N-U. Whole mount in situ hybridization of day 5 embryos with an anti-sense RAG1 probe $(\mathrm{O}, \mathrm{Q})$ and a sense control $(\mathrm{N}, \mathrm{P})$; or with an anti-sense RAG2 probe $(\mathrm{S}, \mathrm{U})$ and a sense control $(\mathrm{R}, \mathrm{T})$. Both lateral $(\mathrm{N}, \mathrm{O}, \mathrm{R}$ and $\mathrm{S})$ and dorsal views $(\mathrm{P}, \mathrm{Q}$, T and U) were presented. Scale bars $=200 \mu \mathrm{m}$ 

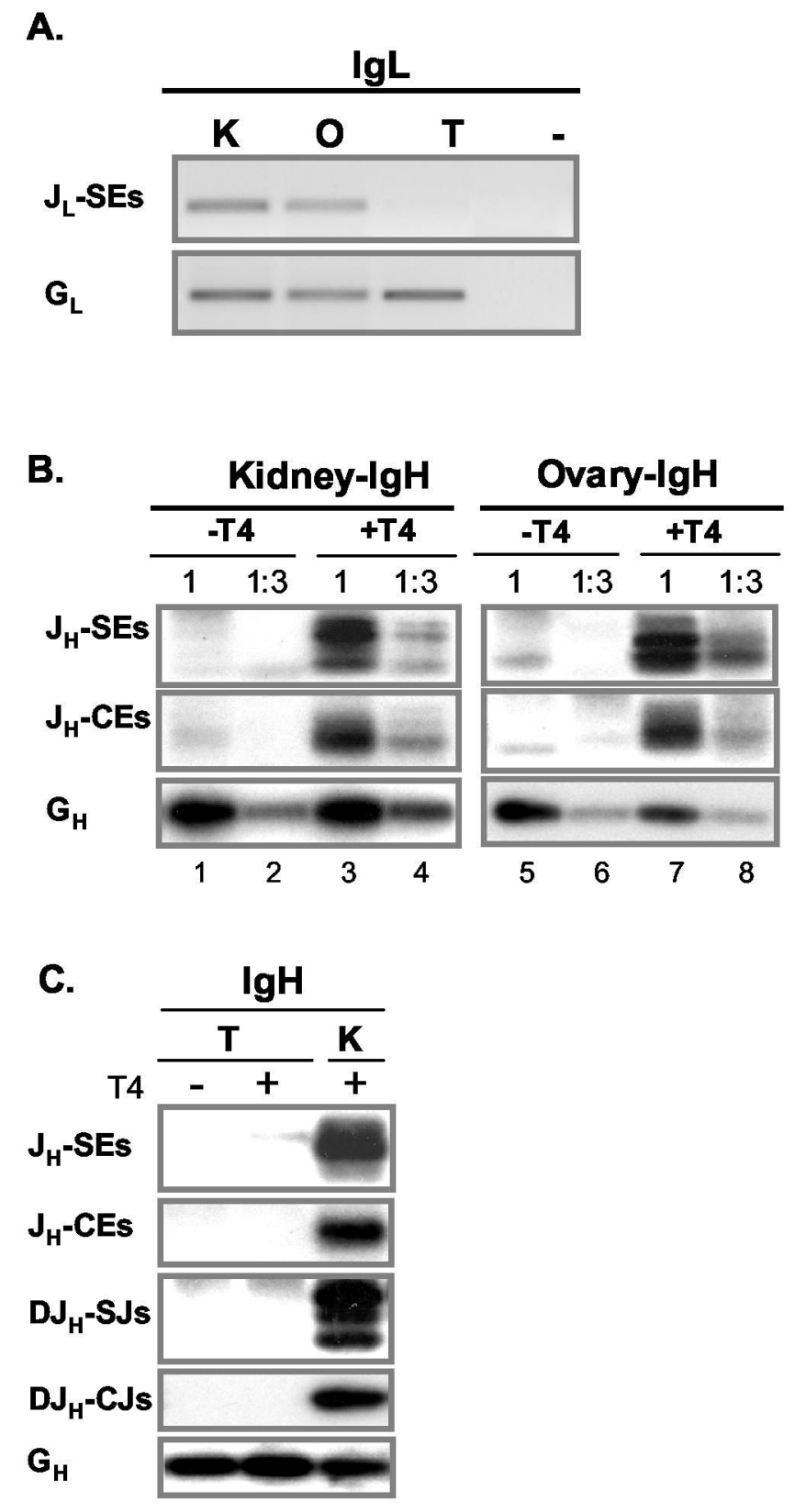

Fig. 2.

Examination of recombination ends in the zebrafish ovary and testis. A. Analysis of recombination ends made at $\operatorname{IgL}$ locus in zebrafish kidney $(\mathrm{K})$, ovary $(\mathrm{O})$ and testis $(\mathrm{T})$. Genomic DNA samples were embedded in DNA-plugs followed by a semi-nested LM-PCR analysis to detect $\mathrm{J}_{\mathrm{L}}-\mathrm{SEs}$. Germline light chain $\left(\mathrm{G}_{\mathrm{L}}\right)$ was included as a control for DNA input. B. Comparison of recombination ends made in the ovary and kidney. Genomic DNA-plugs were treated with or without T4-DNA-POL before analysis by LM-PCR. A semi-quantitative LM-PCR (two different dilutions: 1 and 1:3) followed by Southern blot analysis was performed to detect $\mathrm{J}_{\mathrm{H}^{-}} \mathrm{SEs}, \mathrm{J}_{\mathrm{H}^{-}} \mathrm{CEs}$ and Germline heavy chain $\left(\mathrm{G}_{\mathrm{H}}\right)$ as a control. C. Examination of recombination intermediates and products in zebrafish testis (T). Genomic DNA was treated with or without T4-DNA-POL as indicated, and then Southern blot analysis was performed to 
detect $\mathrm{J}_{\mathrm{H}^{-}} \mathrm{SEs}, \mathrm{J}_{\mathrm{H}^{-}} \mathrm{CEs}, \mathrm{DJ}_{\mathrm{H}^{-}} \mathrm{SJ}$, $\mathrm{DJ}_{\mathrm{H}^{-}}-\mathrm{CJ}$ s and $\mathrm{G}_{\mathrm{H}}$ as a loading control. T4-DNA-POL treated DNA sample from zebrafish kidney was used as a positive control. 

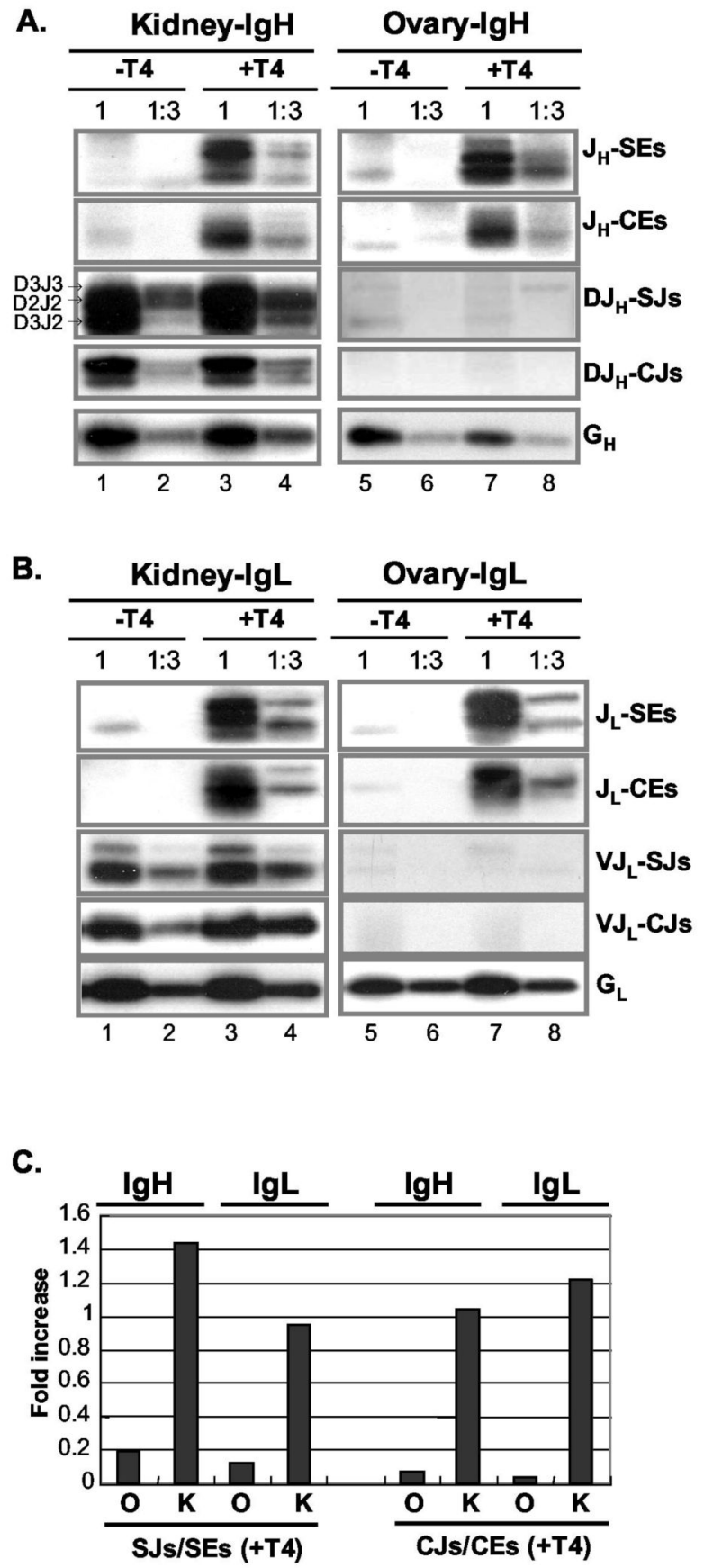

Fig. 3.

Comparison of recombination intermediates and products made in the zebrafish ovary and kidney. A-B. Semi-quantitative LM-PCR and PCR were performed to detect recombination intermediates and products made at $\operatorname{IgH}$ locus (A.) and $\operatorname{IgL}$ locus (B.) in zebrafish ovary $(\mathrm{O})$ and kidney (K). The details are given in (Fig.2B). C. The intensity of PCR bands (T4-DNA-POL treated condition) was analyzed by PhosphoImager and quantified by Image Quant software (Molecular Dynamics). The average ratios of signal joints to signal ends (SJs/SEs) or coding joints to coding ends (CJs/CEs) were derived from both dilutions, and compared between the ovary $(\mathrm{O})$ and the kidney $(\mathrm{K})$. 

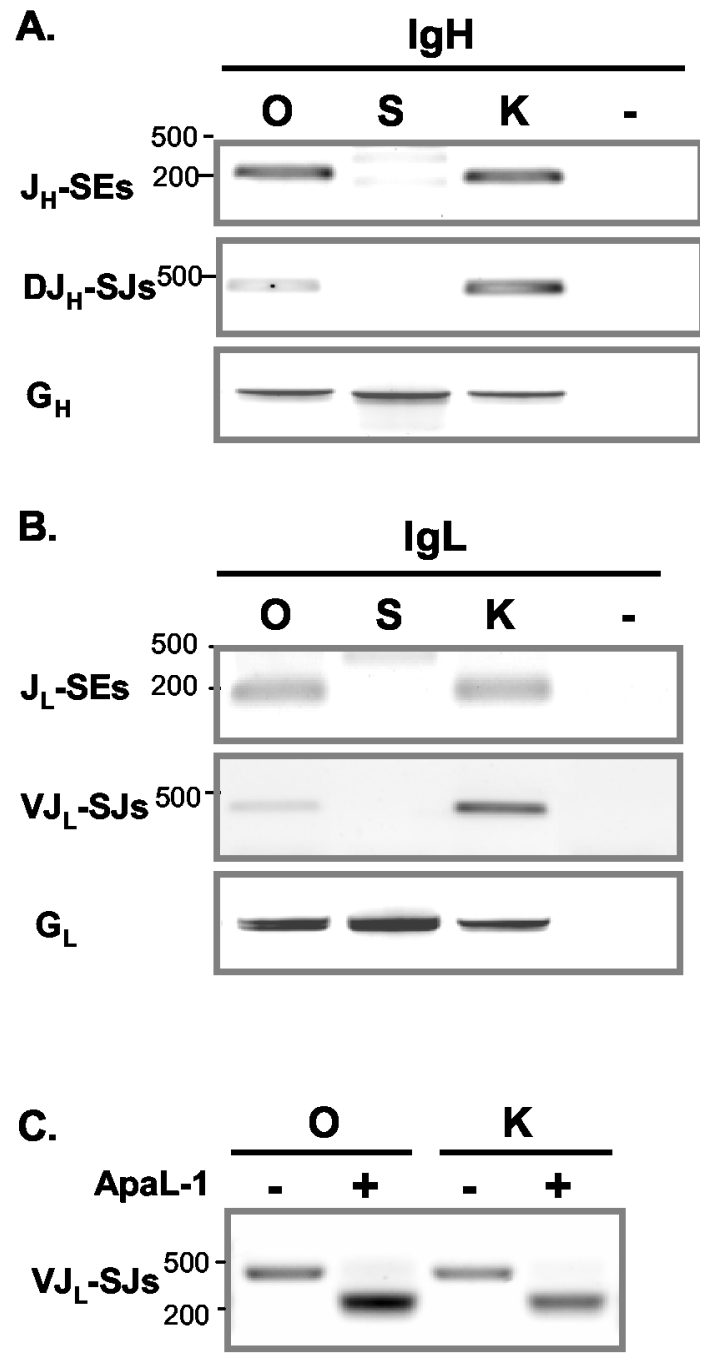

Fig. 4.

Analyses of recombination intermediates and products in the ovary and kidney. A. Analyses of $\mathrm{IgH}$ rearrangement in the ovary $(\mathrm{O})$, the spleen $(\mathrm{S})$ and the kidney $(\mathrm{K})$ by a seminested PCR. $\mathrm{J}_{\mathrm{H}^{-}} \mathrm{SEs}, \mathrm{DJ}_{\mathrm{H}^{-}} \mathrm{SJ}$, and $\mathrm{G}_{\mathrm{H}}$ were presented along with molecular marker for the size (bps). Primers used were listed in Table 1 and 2. locations of each primer were presented in supplemental Figure 1B. B. Detection of $J_{L}-S E s, V_{L}-S J s$ and $G_{L}$ by a semi-nested PCR. C. Examination of fidelity of SJs by ApaL1 digestion. The PCR products amplified from IgLchain $\mathrm{VJ}_{\mathrm{L}}-\mathrm{SJ}$ s that contain ApaL1 site were treated with or without restriction endonuclease ApaL1 overnight, and analyzed by gel electrophoresis. The down-shift of PCR products indicates a digestion by ApaL1, reflecting the presence of the perfect junction of SJs. 
Table 1

Primers used for PCR amplification of Ig recombination ends and joints

\begin{tabular}{|c|c|c|}
\hline Primer & Primer Sequence & DNA accession No. \\
\hline RAG1-5' & 5'-AATCCCAGACTGGAAGCCC-3' & DRU71093 \\
\hline RAG1-3' & 5'-CACACTGCAGGATGCAGACC-3' & DRU71093 \\
\hline RAG2-5' & 5'-ACGCTCATGTCCAACTGGGATA-3' & DRU71094 \\
\hline RAG2-3' & 5'-CTCTGCTGTCTACGCTCAACATGTA-3' & DRU71094 \\
\hline$\beta$-actin-5' & 5'-CGTGACATCAAGGAGAAGCT-3' & AF057040 \\
\hline$\beta$-actin-3' & $5^{\prime}$-TCCTTCTGCATACGGTCAGC-3' & AF057040 \\
\hline 261 & 5'-TTCCTGGACTTTGCATTGCACAA-3 & BX510335 \\
\hline 393 & 5'-GACAAGTCACTGTGACTACTAC-3' & BX510335 \\
\hline 409 & 5'-TGACAGTTTCCTCAGGTAAGACG-3' & BX510335 \\
\hline 262 & 5'-AATCCATGCAGGAGGAGCCGATT-3' & BX510335 \\
\hline 392 & 5'-CTGACGAGACGGTGACCATAGT-3' & BX510335 \\
\hline 279 & 5'-AGTGACATCAGGTAAGCAGTTTCCT-3' & BX510335 \\
\hline 295 & 5'-TGGCTTAGTTGTAAAGATCCATAGC-3' & BX510335 \\
\hline 290 & 5'-TCTGCTGTCAACTATCTGGCT-3' & BX510335 \\
\hline 410 & 5'-GTGAGATTGACACTAATGAAGCC-3' & AF246162 \\
\hline 406 & 5'-GTCTCTACAGTCTCTCTCAGCTGC-3' & AF246162 \\
\hline 404 & 5'-CTATGGATCTGGTTTCTCTGC-3' & AF246162 \\
\hline 267 & 5'-ACCGATCAGTCTGGATGTTCTG-3' & AF246162 \\
\hline 273 & 5'-TACAATGACTTGACCAGCCCAGT-3' & UCSC Genome \\
\hline 274 & 5'-TGGGGAAAACATGACTAACAAAGCA-3' & UCSC Genome \\
\hline 25 & 5'-GCTATGTACTACCCGGGAATTCGTG-3' & (linker-top) \\
\hline 116 & $5^{\prime}-\mathrm{CACGAATTCCC}-3^{\prime}$ & (linker-bottom) \\
\hline
\end{tabular}




\section{Table 2}

PCR primer pairs in end/joint detection

\begin{tabular}{|c|c|c|c|}
\hline \multirow{2}{*}{ Ends/joints } & \multirow{2}{*}{$\begin{array}{l}1^{\text {st }} \text { PCR } \\
\text { primers }\end{array}$} & \multicolumn{2}{|c|}{$2^{\text {nd }}$ PCR } \\
\hline & & primers & sizes \\
\hline $\mathrm{J}_{\mathrm{H}}-\mathrm{SE}$ & $393 / 25$ & $409 / 25$ & $263 \mathrm{bps}$ \\
\hline $\mathrm{J}_{\mathrm{H}^{-}} \mathrm{CE}$ & $\underline{290 / 25}$ & - & \\
\hline $\mathrm{DJ}_{\mathrm{H}^{-}}-\mathrm{SJ}$ & $\underline{409 / 262}$ & $409 / 261$ & $473 \mathrm{bps}$ \\
\hline $\mathrm{DJ}_{\mathrm{H}}-\mathrm{CJ}$ & $\overline{295 / 290}$ & & \\
\hline $\mathrm{G}_{\mathrm{H}}$ & $279 / 392$ & - & \\
\hline $\mathrm{J}_{\mathrm{L}}-\mathrm{SE}$ & $410 / 25$ & $\underline{406 / 25}$ & $280 \mathrm{bps}$ \\
\hline $\mathrm{J}_{\mathrm{L}}-\mathrm{CE}$ & $\underline{273 / 25}$ & $\overline{-}$ & \\
\hline $\mathrm{VJ}_{\mathrm{L}}-\mathrm{SJ}$ & $\overline{410 / 267}$ & $\underline{406 / 267}$ & $464 \mathrm{bps}$ \\
\hline $\mathrm{VJ}_{\mathrm{L}}-\mathrm{CJ}$ & $\underline{404 / 274}$ & - & \\
\hline $\mathrm{G}_{\mathrm{L}}$ & $\overline{404 / 274}$ & - & \\
\hline
\end{tabular}

Underlined primer pairs were used for semi-quantitative PCR followed by Southern blot analyses. 
Table 3

Structure of individual signal ends made at $\mathrm{J}_{\mathrm{H}}$ and $\mathrm{J}_{\mathrm{L}}$ loci of the ovary

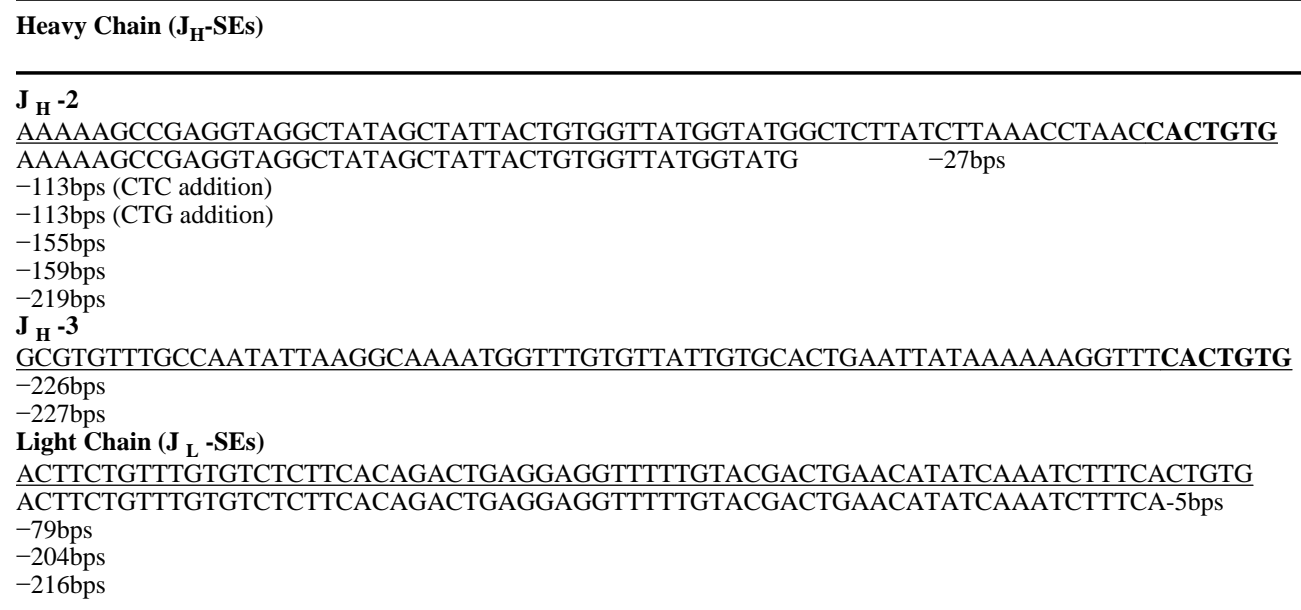

Signal end sequences are aligned to the germline J gene segments (underlined). The extent of nucleotide loss is indicated by the - number. The heptamer of recombination signal sequence is highlighted as bold. 